\title{
Maximal cluster sets on spaces of holomorphic functions
}

\author{
José A. Prado-Bassas
}

Received: Month-1 99, 2003; Accepted: Month-2 99, 2004

\begin{abstract}
Summary: This is an expository paper where we relate some aspects of the problem of looking for holomorphic functions with maximal cluster sets under the action of operators defined on spaces of holomorphic functions. Some functional generalizations of cluster sets, as well as special spaces of analytic functions, are also considered.
\end{abstract}

\section{Introduction and Notation}

In this paper, which has an expository nature, we consider the so called cluster sets, that is the set of accumulation points of the set of values taken by a (usually, holomorphic) function on prescribed subsets. We are specially interested in the action of holomorphic operators in order to obtain maximal cluster sets, see below for details. Extensions of classical cluster sets, where they are replaced by adequate families of functions, as well as special spaces of analytic functions (such as Hardy or Bergman spaces) are also included in this survey. Firstly, we fix some notation.

Throughout this paper, $\mathbb{Z}$ will stand for the set of all integers, $\mathbb{N}$ is the set of positive integers, $\mathbb{N}_{0}:=\mathbb{N} \cup\{0\}, \mathbb{C}$ is the complex plane and $\widehat{\mathbb{C}}$ is its one-point compactification or Riemann sphere $\mathbb{C} \cup\{\infty\}$. We denote by $\mathbb{D}$ the open unit disk $\{z \in \mathbb{C}:|z|<1\}$ and by $\mathbb{T}$ the unit circle $\{z \in \mathbb{C}:|z|=1\}$. If $A \subset \mathbb{C}$ then $A^{\prime}, A^{\circ}$ and $\partial A$ represent its set of accumulation points, its interior and its boundary in $\widehat{\mathbb{C}}$, respectively. Also, if $F: A \rightarrow \mathbb{C}$ is a function on $A$ then $\|F\|_{A}:=\sup _{z \in A}|F(z)|$. Moreover, if $G$ is a domain (:= connected, nonempty open subset) of $\mathbb{C}$, then $H(G)$ will stand for the space of holomorphic functions on $G$. It becomes a completely metrizable space (hence a Baire space) when it is endowed with the compact open topology (see [25, pages 238-239]). The symbol $\mathcal{K}(G)$ will stand for the family of all compact subsets of $G$ while $\mathcal{K}_{C}(G)$ is the family of compact subsets $K$ of $G$ such that $\mathbb{C} \backslash K$ is connected. A Jordan domain is a domain $G$ of $\mathbb{C}$ whose boundary in $\widehat{\mathbb{C}}$ is a topological image of $\mathbb{T}$, and a simply connected domain is a domain $G$ whose complement in $\widehat{\mathbb{C}}$ is also connected. Finally, if

AMS 2000 subject classification: Primary: 30D40; 30-02 Secondary: 30E10; 47B38

Key words and phrases: maximal cluster sets, functional cluster sets, holomorphic monster, $T$-monster, strong omnipresence, total omnipresence, MRCS-property 
$K \subset \mathbb{C}$ is compact, we denote by $\mathcal{A}(K)$ the family of all continuous functions on $K$ that are holomorphic in $K^{\circ}$.

If $G$ is a domain in $\mathbb{C}$, then a curve in $G$ tending to the boundary (notation: $\gamma \rightarrow \partial G$ ) is a continuous map $\gamma:[0,1) \rightarrow G$ such that for each compact set $K \subset G$ there is $u_{0}=u_{0}(K) \in[0,1)$ with $\gamma(u) \in G \backslash K$ for all $u>u_{0}$ (in particular if $G=\mathbb{D}$ then $\gamma$ tends to the boundary $\mathbb{T}$ if and only if $\lim _{u \rightarrow 1^{-}}|\gamma(u)|=1$ ). By abuse of language, we will usually make the identification $\gamma:=\gamma([0,1))$.

Finally if $A$ is a subset of a topological space $X$, we say that $A$ is residual if its complement is of Baire first category, or equivalently, if it contains a $G_{\delta}$-dense set (cf. [36]).

The notion of cluster set was first introduced by P. Painlevé [37] as a tool to study intuitively the behavior of an analytic function around one of its singularities. Although we are considering only the case of analytic functions, this is not a real restriction, because the notion of cluster set only depends of the existence of a limit. For essential background on cluster sets the reader is referred to the surveys [18] and [35] by CollingwoodLowhater and Noshiro, respectively. Let us recall some notions.

Definition 1.1 Let $G$ be a domain of $\mathbb{C}, F: G \rightarrow \mathbb{C}$ be a function and $A$ be a subset of $G$ with $A^{\prime} \cap \partial G \neq \varnothing$. The cluster set of $F$ along $A$ is the set

$$
\begin{aligned}
C_{A}(F)=\{w \in \widehat{\mathbb{C}}: & \text { there exists a sequence }\left\{z_{n}\right\}_{n=1}^{\infty} \subset A \text { tending to } \\
& \text { some point of } \left.\partial G \text { such that } \lim _{n \rightarrow \infty} F\left(z_{n}\right)=w\right\} .
\end{aligned}
$$

Moreover, if $t_{0} \in A^{\prime} \cap \partial G$, then the cluster set of $F$ along $A$ at $t_{0}$ is the set

$$
\begin{gathered}
C_{A}\left(F, t_{0}\right)=\{w \in \widehat{\mathbb{C}}: \\
\text { there exists a sequence }\left\{z_{n}\right\}_{n=1}^{\infty} \subset A \text { tending to } t_{0} \\
\text { such that } \left.\lim _{n \rightarrow \infty} F\left(z_{n}\right)=w\right\} .
\end{gathered}
$$

It is clear that both $C_{A}(F)$ and $C_{A}\left(F, t_{0}\right)$ are closed subsets of $\widehat{\mathbb{C}}$ and that $C_{A}(F)$ is the $\widehat{\mathbb{C}}$-closure of $\bigcup_{t \in \partial G} C_{A}(F, t)$. If $A=G$ then the subscript " $A$ " and the expression "along $A$ " are usually omitted and we speak about the global cluster set. A special important case occurs when $G=\mathbb{D}, t_{0} \in \mathbb{T}$ and $A$ is the radius $A:=\left\{u t_{0}: u \in[0,1)\right\}$; then the radial cluster set is defined as the set $C_{\varrho}\left(F, t_{0}\right):=C_{A}(F)=C_{A}\left(F, t_{0}\right)$. We will say that a cluster set is maximal when it is equal to $\widehat{\mathbb{C}}$.

Historically, the problem of looking for holomorphic functions with maximality properties of cluster sets has been of interest for mathematicians. Observe that, if $z_{0}$ is an isolated essential singularity of a holomorphic function $f$, then the cluster set $C\left(f, z_{0}\right)$ is maximal due to the Casorati-Weierstrass Theorem (see [1]). This is a first example of finding functions with (one) maximal cluster set, but it does not allow to prefix how to approach the boundary, in other words, it only tells about global cluster sets.

An example of the above situation but prescribing a set to approach $\partial G$ can be easily obtained thanks to the next well known Weierstrass' interpolation theorem, cf. [38, Theorem 15.13].

Theorem 1.2 Let $G \subset \mathbb{C}$ be a domain, $\left(a_{n}\right)_{n} \subset G$ be a sequence in $G$ of distinct points without accumulation points in $G$ and $\left(\omega_{n}\right)_{n} \subset \mathbb{C}$ be any complex sequence. Then there is a function $f \in H(G)$ such that $f\left(a_{n}\right)=\omega_{n}$ for all $n \in \mathbb{N}$. 
As a consequence, if we fix a non-relatively compact subset $A$ of $G$, then we can find a sequence $\left(a_{n}\right)_{n} \subset A$ satisfying the hypothesis of the last theorem. By choosing as $\left(w_{n}\right)_{n}$ an enumeration of the set $\mathbb{Q}[i]:=\mathbb{Q}+i \mathbb{Q}$ of rational complex numbers, we obtain a function $f \in H(G)$ such that $C_{A}(f)$ is maximal. If we also fix a boundary point $t_{0} \in A^{\prime} \cap \partial G$ (that is, accessible through $A$ ), a similar reasoning gives us a function $f \in H(G)$ with $C_{A}\left(f, t_{0}\right)=\widehat{\mathbb{C}}$.

At this point, several questions arise. In this survey we will be interested in studying the "size" (in a topological or algebraic sense) of functions with maximality properties of their cluster sets as well as in looking for functions with maximality properties at every boundary point or along suitable paths. We will be specially concerned in looking for functions such that their images under the action of operators present maximality properties for cluster sets, and in some "functional" generalizations of the concept of cluster set.

\section{Operators generating maximal cluster sets}

\subsection{Maximal global cluster sets}

The last result of the preceding section allows us to obtain holomorphic functions with maximal cluster sets along any prefixed non-relatively compact subset of $G$. But it is also interesting to study the topological size of the set of functions with this properties, specifically the size of the set of functions with this property at "many" boundary points and even such that their derivatives have also maximal cluster sets. Following this line, in 1992, Bernal [4] introduced the concept of (holomorphic) omnipresent operator in the following way.

Definition 2.1 Let $G$ be a domain of $\mathbb{C}$ and $T: H(G) \rightarrow H(G)$ be a (not necessarily linear) continuous operator. We say that $T$ is omnipresent if and only if the set

$$
\{f \in H(G): C(T f, t)=\widehat{\mathbb{C}} \text { for all } t \in \partial G\}
$$

is residual.

It is also showed in [4] -by using Cauchy's formula for derivatives, together with Runge's approximation theorem and Mergelyan's approximation theorem (see [21])- that the derivative operator of any order $m, D^{m}: f \mapsto f^{(m)}$, and the antiderivative operator of any order $m, D^{-m}: f \mapsto f^{(-m)}$ (in the case of a simply connected domain $G$ and for any $m \in \mathbb{N}, f^{(-m)}$ is defined by fixing a point $a \in G$ and saying that $f^{(-m)}$ is the unique function $F \in H(G)$ such that $F^{(m)}=f$ and $F^{(h)}(a)=0$ for $\left.h=0,1, \ldots, m-1\right)$ are omnipresent, so given a simply connected domain $G \subset \mathbb{C}$ and any $j \in \mathbb{Z}$, the residuality of the set

$$
\left\{f \in H(G): C\left(f^{(j)}, t\right)=\widehat{\mathbb{C}} \text { for all } t \in \partial G\right\}
$$

is provided. But thanks to Baire's Theorem we get the next result (see also [31] and [23, Kapitel 3]).

Theorem 2.2 Assume that $G$ is a domain of $\mathbb{C}$. We have: 
(a) The set

$$
\left\{f \in H(G): C\left(f^{(j)}, t\right)=\widehat{\mathbb{C}} \text { for all } t \in \partial G \text { and all } j \in \mathbb{N}_{0}\right\}
$$

is residual in $H(G)$.

(b) If $G$ is simply connected, then the set

$$
\left\{f \in H(G): C\left(f^{(j)}, t\right)=\widehat{\mathbb{C}} \text { for all } t \in \partial G \text { and all } j \in \mathbb{Z}\right\}
$$

is residual in $H(G)$.

The above theorem deals with the maximality of global cluster sets of holomorphic functions. Later, in 1995 the same author [5] extended this result by prefixing where to approach the boundary in the case of derivative operators (in turn, Calderón [16, Theorems 2.1 and 3.5] extended it to an infinite order differential operator).

Theorem 2.3 Let $G \subset \mathbb{C}$ be a domain. Given any non-relatively compact subset $A$ of $G$, the set

$$
\left\{f \in H(G): C_{A}\left(f^{(n)}\right)=\widehat{\mathbb{C}} \text { for all } n \in \mathbb{N}_{0}\right\}
$$

is residual in $H(G)$.

Now, if we fix a boundary point $t_{0} \in A^{\prime} \cap \partial G$, we get also the residuality of the set $\left\{f \in H(G): C_{A}\left(f^{(n)}, t_{0}\right)\right.$ for all $\left.n \in \mathbb{N}_{0}\right\}$. Observe that, as we said before, this result allows us to prescribe how the sequence reaches the boundary (through a segment, a sector, a curve, etc) while with the omnipresence we cannot make this restriction.

As a direct consequence of Theorem 2.3, if we prefix any countable set $\Gamma$ of curves in $G$ tending to the boundary, the Baire Theorem provides a residual subset $M$ of $H(G)$ such that $C_{\gamma}\left(f^{(j)}\right)=\widehat{\mathbb{C}}$ for all integer $j \geq 0$, all $\gamma \in \Gamma$ and all $f \in M$. In particular, if $G=\mathbb{D}$ and we prefix a dense and countable subset $\Lambda \subset \mathbb{T}$ we get the residuality of the set $\left\{f \in H(\mathbb{D}): C_{\varrho}\left(f^{(j)}, t\right)=\widehat{\mathbb{C}}\right.$ for all $t \in \Lambda$ and all $\left.j \geq 0\right\}$. Analogously, if for every boundary point $t \in \Lambda$ we fix a sequence $\left(a_{n}^{(t)}\right)_{n} \subset G$ such that $a_{n}^{(t)} \rightarrow t(n \rightarrow \infty)$, then we get the existence of a residual set of functions $f \in H(G)$ with $C_{\left(a_{n}^{(t)}\right)_{n}}(f, t)=\widehat{\mathbb{C}}$ for all $t \in \Lambda$. Observe that in this situation, if $G=\mathbb{D}$, we can prefix sequences with tangential (non-tangential, respectively) approximation to any boundary point of $\Lambda$.

\subsection{Maximal radial cluster sets}

This subsection is devoted to describe the problem of maximal radial cluster sets in $\mathbb{D}$, mainly under the action of operators on holomorphic functions. In this direction, R. Tenthoff [40] in 2000 proved, among other results, the next theorem about maximality of radial cluster sets.

Theorem 2.4 There is a dense subset $\mathcal{D} \subset H(\mathbb{D})$ such that for every $f \in \mathcal{D}$, every integer number $j \in \mathbb{Z}$, and every boundary point $t \in \mathbb{T}, C_{\varrho}\left(f^{(j)}, t\right)=\widehat{\mathbb{C}}$. 
By using the Osgood-Carathéodory theorem on continuous extensions of isomorphisms up to the boundary (see [24]), Theorem 2.4 can be extended to any Jordan domain $G$ of $\mathbb{C}$ in this way. Given any Jordan domain $G$ there is a family $\Gamma=\left\{\gamma_{t}: t \in \partial G\right\}$ of curves in $G$ with $\gamma_{t} \rightarrow t$ for all $t \in \partial G$, satisfying that the set of functions $f \in H(G)$ such that for every $j \in \mathbb{Z}$ and every $t \in \partial G$, the cluster set $C_{\gamma_{t}}\left(f^{(j)}, t\right)$ is maximal, is dense in $H(G)$.

In view of this result, one may ask about the residuality of the set of functions $f \in$ $H(\mathbb{D})$ such that for each $j \in \mathbb{Z}$ and all $t \in \mathbb{T}$, the equality $C_{\varrho}\left(f^{(j)}, t\right)=\widehat{\mathbb{C}}$ holds.

A first attempt in this direction can be derived of a statement of Kierst and Szpilrajn (cf. [28, Section 4]). In particular, they proved the residuality of the set of holomorphic functions in $\mathbb{D}$ with maximal radial cluster set at any boundary point.

A recent statement by Bernal, Calderón and the author [13, Theorem 2.2] (see Theorem 2.7 below) gives a partial answer to the above question. But we first need two definitions.

Definition 2.5 Let $G \subset \mathbb{C}$ be a domain and $T: H(G) \rightarrow H(G)$ be a (not necessarily linear) continuous operator. We say that $T$ is locally stable near the boundary if for each $K \in \mathcal{K}(G)$ there exists a compact subset $M \in \mathcal{K}(G)$ such that for each compact subset $L \in \mathcal{K}_{C}(G)$ with $L \subset G \backslash M$, each function $f \in H(G)$ and each positive number $\varepsilon>0$, there exist a compact subset $L^{\prime} \in \mathcal{K}_{C}(G)$ with $L^{\prime} \subset G \backslash K$ and a positive number $\delta>0$ such that

$$
\left[g \in H(G) \text { and }\|f-g\|_{L^{\prime}}<\delta\right] \text { implies }\|T f-T g\|_{L}<\varepsilon \text {. }
$$

Definition 2.6 Let $G \subset \mathbb{C}$ be a domain, $T: H(G) \rightarrow H(G)$ be a (not necessarily linear) continuous operator and $\mathcal{A} \subset H(G)$. We say that $T$ has locally dense range at $\mathcal{A}$ near the boundary if there exists a compact subset $S \in \mathcal{K}(G)$, such that for each $f \in \mathcal{A}$, each compact subset $L \in \mathcal{K}_{C}(G)$ with $L \subset G \backslash S$ and each positive number $\varepsilon>0$, there is a function $F \in H(G)$ such that $\|T F-f\|_{L}<\varepsilon$.

We are now able to state the promised result. Again, Runge's theorem is an essential tool in the proof.

Theorem 2.7 Let $T$ be a locally stable continuous operator on $H(\mathbb{D})$ with locally dense range at the constant functions near the boundary. Then the set $\mathcal{M}_{\varrho}(T):=\{f \in H(\mathbb{D})$ : $C_{\varrho}(T f, t)=\widehat{\mathbb{C}}$ for all $\left.t \in \mathbb{T}\right\}$ is dense in $H(\mathbb{D})$.

An operator $T$ on $H(\mathbb{D})$ with the property that $\mathcal{M}_{\varrho}(T)$ is dense in $H(\mathbb{D})$ is called to have the maximal radial cluster set property (in short, MRCS property). In particular, every derivative operator (including the identity operator $I$ ) satisfies the MRCS property. Hence we get the next corollary just by taking into account that $\mathcal{M}_{\varrho}(T)$ is always a $G_{\delta}$-subset.

Corollary 2.8 There exists a residual set of functions $f \in H(\mathbb{D})$ such that the cluster set $C_{\varrho}\left(f^{(j)}, t\right)$ is maximal for all $t \in \mathbb{T}$ and all $j \geq 0$. 
Observe that the antiderivative operator of any order is not locally stable, so we cannot apply Theorem 2.7 and the problem of the residuality of the set $\{f \in H(\mathbb{D})$ : $C_{\varrho}\left(f^{(j)}, t\right)=\widehat{\mathbb{C}}$ for all $t \in \mathbb{T}$ and all $\left.j \in \mathbb{Z}\right\}$ is still open.

In the same work -see [13, Section 3]- Bernal, Calderón and the author also proved that many classical operators, including composition, multiplication, superposition operators (under suitable conditions) and non-zero infinite order differential operators have the MRCS property. But before state the result, let us define precisely the operators we need.

Definition 2.9 Let $G$ be a domain in $G$

1. Let $\Phi(z)=\sum_{k \geq 0} \phi_{k} z^{k}$ be an entire function of subexponential (resp. exponential) type, that is, for all number $\varepsilon>0$ there is a constant $A=A(\varepsilon)>0$ such that $|\Phi(z)| \leq A e^{\varepsilon|z|}$ (resp. there are positive constants $A$ and $B$ such that $|\Phi(z)| \leq$ $\left.A e^{B|z|}\right)$, or equivalently, $\lim _{k \rightarrow \infty}\left(k !\left|\phi_{k}\right|\right)^{1 / k}=0\left(\right.$ resp. $\limsup _{k \rightarrow \infty}\left(k !\left|a_{k}\right|\right)^{1 / k}<$ $+\infty)$, then $\Phi(D):=\sum_{k \geq 0} \phi_{k} D^{k}$ defines a linear continuous operator on $H(\mathbb{D})$ (actually on $H(G)$ with $G$ any domain in $\mathbb{C}$ ) called infinite order differential operator, where $D^{0}:=$ the identity operator (see $[3, \S 6.4]$ ).

2. Let $\psi \in H(G)$. We define the multiplication operator on $H(G)$ as follows:

$$
M_{\psi}: f \in H(G) \mapsto \psi \cdot f \in H(G) .
$$

3. Let $H(G, G)=\{g \in H(G): g(G) \subset G\}$. Given $\varphi \in H(G, G)$ we define the (right) composition operator $C_{\varphi}$ as

$$
C_{\varphi}: f \in H(G) \mapsto f \circ \varphi \in H(G) .
$$

4. Let $\varphi$ be an entire function. The superposition operator on $H(G)$ is defined as

$$
L_{\varphi}: f \in H(G) \mapsto \varphi \circ f \in H(G) .
$$

We are now able to present the result, that collects the main examples of operators with the MRCS-property.

\section{Theorem 2.10}

1. Let $\Phi \in H(\mathbb{C})$ be a nonzero entire function of subexponential type. Then the associated differential operator $\Phi(D)$ has the MRCS-property.

2. Let $\psi \in H(\mathbb{D})$ and $Z(\psi)$ be the set of zeros of $\psi$. If $Z(\psi)$ is finite then the multiplication operator $M_{\psi}$ has the MRCS-property.

3. Let $\varphi \in H(\mathbb{D})$ be proper, that is, the preimage of every compact is also compact. Then the composition operator $C_{\varphi}$ has the MRCS-property.

4. Let $\varphi$ be an entire function. Then the superposition operator $L_{\varphi}$ generated by $\varphi$ has the MRCS-property if and only if $\varphi$ is non constant.

5. Let $T, S: H(\mathbb{D}) \rightarrow H(\mathbb{D})$ be operators such that $T$ has the MRCS-property and $S$ is linear and onto. Then $T \circ S$ has the MRCS-property. In particular, every linear and onto operator has the MRCS-property. 


\subsection{Maximal cluster sets along curves}

Going back to cluster sets along arbitrary curves in general domains, we find the next statement, which can be derived from an old, not very known result due to Kierst and Szpilrajn [28, Section 4].

Theorem 2.11 The set of functions $f \in H(\mathbb{D})$ such that $C_{\gamma}(f, t)=\widehat{\mathbb{C}}$ for each $t \in \mathbb{T}$ and all curve $\gamma \subset \mathbb{D}$ with $\gamma \rightarrow \mathbb{T}$ and disjoint of some segment-depending on $\gamma$ - in $\mathbb{D}$ ending at $\mathbb{T}$ is residual in $H(\mathbb{D})$.

The condition on each curve $\gamma$ given in Theorem 2.11 is fulfilled (for $G=\mathbb{D}$ ) if $\gamma$ is an admissible curve. This means that the set of oscillation values (or $\omega$-limit) of a curve $\gamma \subset G$ with $\gamma \rightarrow \partial G$, given by

$$
\operatorname{Osc}(\gamma):=\left\{t \in \partial G: \text { exists }\left(z_{n}\right)_{n} \subset \gamma \text { with } \lim _{n \rightarrow \infty} z_{n}=t\right\}
$$

is non-total, that is, $\operatorname{Osc}(\gamma) \neq \partial G$, or equivalently, $\gamma^{\prime} \cap \partial G \neq \partial G$.

Recently, in 2002, A. Boivin, P. M. Gauthier and P. V. Paramonov [14, Theorem 5] have shown a result on elliptic differential operators $L$ on $\mathbb{R}^{N}$. In the special case of the Cauchy operator $L=\bar{\partial}=\frac{1}{2}\left(\partial_{x}+i \partial_{y}\right)$ (with $N=2$ ), such result reads as follows.

Theorem 2.12 Let $G$ be a domain in $\mathbb{C}$ such that its boundary $\partial G$ has no connected component consisting of a single point. Then there exists a function $f \in H(G)$ with the property that for each point $t \in \partial G$, for each curve $\gamma \subset G$ ending at $t$ and each $j \in \mathbb{N}_{0}$, the cluster set $C_{\gamma}\left(f^{(j)}, t\right)$ is maximal.

It is also observed in [14] that this result is close to being sharp. Indeed, it is shown in [14, Proposition 4] that if $\partial G$ has an isolated point $b$, then for each function $f \in H(G)$, there exists a curve $\gamma \subset G$ ending at $b$ such that $C_{\gamma}(f)$ collapses to a single point.

The following theorem due to the Bernal, Calderón and the author [12] extends the result of Kierst and Szpilrajn by setting the large "algebraic" (not only "topological") size of the set of functions $f \in H(\mathbb{D})$ with maximal cluster set along any admissible curve. The Nersesjan approximation theorem (see [21] and [34]) is an important tool in its proof.

Theorem 2.13 Let $G$ be a Jordan domain. Then there is a dense linear manifold $\mathcal{D} \subset$ $H(G)$ such that for every $f \in \mathcal{D} \backslash\{0\}$ and every admissible curve $\gamma \subset G$ we have $C_{\gamma}(f)=\widehat{\mathbb{C}}$.

A natural question arising in this context is whether one can replace the (almost) arbitrary curve $\gamma$ to an arbitrary sequence $\left\{z_{n}\right\}_{n=1}^{\infty}$ tending to the boundary. But the next assertion (see [12, Proposition 2.2]) answers it in the negative.

Proposition 2.14 If $G \subset \mathbb{C}$ is a bounded domain and $f \in H(G)$ then there are a point $t \in \partial G$, a value $A \in \mathbb{C}$ and a sequence $\left\{z_{n}\right\}_{n=1}^{\infty} \subset G$ with $z_{n} \rightarrow t(n \rightarrow \infty)$ such that $\lim _{n \rightarrow \infty} f\left(z_{n}\right)=A$. 
To end this section, we remark that it is possible to extend the searching of large sets of functions with maximal cluster sets along arbitrary curves to more general settings (see for instance[14] and [6]).

We point out that Theorem 2.13 has been recently extended (see [6]) to domains in $\mathbb{R}^{N}$ having no connected component consisting of a single point, to elliptic differential operators $L$ and to certain class of operators $T$ acting on the space of $L$-analytic functions; the holomorphic case and the operators $T=D^{n}$ are contained as special cases.

\section{Generalized cluster sets}

In this section we are interested in some generalizations of the notion of cluster set. Such generalizations were first introduced by Luh [31]. Let us recall that the classical (global) cluster set of a holomorphic function $f$ on a domain $G$ is the set of complex points $\omega \in \widehat{\mathbb{C}}$ such that there exists a sequence $\left(z_{n}\right)_{n}$ in $G$ tending to some boundary point with $f\left(z_{n}\right) \rightarrow \omega(n \rightarrow \infty)$ (see Definition 1.1).

The generalization proposed by Luh starts with the question of the behavior of such a function $f$, when the sequence $\left(z_{n}\right)_{n}$ occurring above depends slightly on a complex variable $z$. To be precise, the aim of this new notion is to investigate the behavior of the functions $f\left(a_{n} z+b_{n}\right)$ when $z$ belongs to some complex subset $S, a_{n} z+b_{n} \in G$ for all $z \in S$ and $a_{n} z+b_{n}$ tends, in some sense, to some boundary point of $G$. These modified cluster sets introduced by Luh are of the three types given in the next definition (see [31]), and depend on the concept of limit taken in the function space considered.

Definition 3.1 Let $G$ be a domain in $\mathbb{C}$. For each compact subset $K \subset \mathbb{C}$, each open set $U \subset \mathbb{C}$ and each (Lebesgue) measurable set $S \subset \mathbb{C}$, for each boundary point $t$ of $G$, and each function $f \in H(G)$, we define:

- $S(f, G, t, K)$ will be the set of functions $\varphi: K \rightarrow \mathbb{C}$ for which there exists a sequence of linear affine mappings $\tau_{n}(z)=a_{n} z+b_{n}$ such that $\tau_{n}(K) \subset G$ for all $n \in \mathbb{N}, a_{n} \rightarrow 0, b_{n} \rightarrow t(n \rightarrow \infty)$ and $f\left(\tau_{n}(z)\right) \rightarrow \varphi$ uniformly on $K$.

- $R(f, G, t, U)$ will denote the set of functions $\varphi: U \rightarrow \mathbb{C}$ for which there exists a sequence of linear affine mappings $\tau_{n}(z)=a_{n} z+b_{n}$ such that $\tau_{n}(U) \subset G$ for all $n \in \mathbb{N}, a_{n} \rightarrow 0, b_{n} \rightarrow t(n \rightarrow \infty)$ and $f\left(\tau_{n}(z)\right) \rightarrow \varphi$ uniformly on compacta of $U$.

- $T(f, G, t, S)$ will be the set of functions $\varphi: S \rightarrow \widehat{\mathbb{C}}$ for which there exists a sequence of linear affine mappings $\tau_{n}(z)=a_{n} z+b_{n}$ such that $\tau_{n}(S) \subset G$ for all $n \in \mathbb{N}, a_{n} \rightarrow 0, b_{n} \rightarrow t(n \rightarrow \infty)$ and $f\left(\tau_{n}(z)\right) \rightarrow \varphi$ almost everywhere on $S$.

It is quite obvious that $S(f, G, t, K) \subset \mathcal{A}(K), R(f, G, t, U) \subset H(U)$ and that $T(f, G, t, S) \subset M(S):=\{f: S \rightarrow \widehat{\mathbb{C}}: f$ is measurable $\}$. Also in the special case $K=\{0\}=S$, we get $S(f, G, t, K)=C(f, t)=T(f, G, t, S)$, that is, we recover the classical cluster sets.

The first result about maximality of modified cluster sets can be found in [29], where it is showed the existence of a holomorphic function $f$ on $\mathbb{D}$, such that $S(f, \mathbb{D}, t, K)=$ 
$\mathcal{A}(K)$ for all compact set $K \in \mathcal{K}_{C}(\mathbb{C})$. This statement was strongly extended by Luh in [31], as follows.

Theorem 3.2 Let $G \subset \mathbb{C}, G \neq \mathbb{C}$, be an open set with simply connected components. Then there exists a function $f \in H(G)$ such that

(a) $S(f, G, t, K)=\mathcal{A}(K)$ for every compact subset $K \in \mathcal{K}_{C}(\mathbb{C})$.

(b) Each derivative $f^{(j)}(j \in \mathbb{N})$ of $f$ and each antiderivative of $f$ of arbitrary order has the same boundary behavior described in (a).

Functions satisfying (a) and (b) are called holomorphic monsters (in the sense of Luh). In addition, he shows in [31] that the set $\mathcal{M}(G)$ of holomorphic monsters in $G$ is dense in $H(G)$. But this is not the unique wild behavior of holomorphic monsters. In fact, Luh also proved that the maximality of modified cluster sets of the first type, implies the maximality of modified cluster sets of the other types. As in [30], Mergelyan's theorem on polynomial approximation and Lusin's theorem on approximation of measurable functions by continuous ones, are crucial tools.

Theorem 3.3 Let $G \subset \mathbb{C}, G \neq \mathbb{C}$, be an open set with simply connected components, $t \in \partial G$ be a fixed boundary point and $f \in H(G)$ satisfying that $S(f, G, t, K)=\mathcal{A}(K)$ for all compact subset $K \in \mathcal{K}_{C}(\mathbb{C})$. Then the function $f$ has the following properties:

(1) $R(f, G, t, U)=H(U)$ for every bounded open set $U \subset \mathbb{C}$ with simply connected components.

(2) For every bounded measurable set $S \subset \mathbb{C}, T(f, G, t, S)=M(S)$.

In fact, combining the last two theorems, we conclude that if $f$ is a holomorphic monster, then every derivative and every antiderivative of $f$ and the function $f$ itself have maximal modified cluster sets of the three types. Monster with additional properties were also constructed by Luh [32], Schneider [39] (monsters with properties of univalence) and Luh, Martirosian and Müller [33] (monsters with gap power series). Kanatnikov [26, 27] studied in the eighties universal boundary behavior (only of $f$ itself) for meromorphic functions.

In 1987, Grosse-Erdmann [22, Kapitel 3], continued the study of these modified cluster sets and showed that a function $f \in H(G)$ is a holomorphic monster if and only if every derivative and every antiderivative of arbitrary order (say $F$ ) satisfies that for each Jordan domain $\Omega \subset \mathbb{C}$ and each boundary point $t \in \partial G$, the modified cluster set $R(F, G, t, \Omega)$ equals $H(\Omega)$. In fact [22, Satz 3.0.2] it suffices to take $\Omega=\mathbb{D}$. In addition, he also proved, using universality techniques, the residuality of the set $\mathcal{M}(G)$.

Recently, Armitage [2], and Calderón and Müller [17] have shown the existence of harmonic functions with such a wild behavior near the boundary. The last two authors have considered a more general setting, namely, the space of $L$-analytic functions for an elliptic operator $L$.

Following the study of holomorphic monsters, Bernal and Calderón generalized in [7] the concept of monster by introducing holomorphic operators. 
Definition 3.4 Let $G$ be an arbitrary open subset of $\mathbb{C}, G \neq \mathbb{C}$ and let $T: H(G) \rightarrow$ $H(G)$ be a (not necessarily linear) continuous operator. A function $f \in H(G)$ is said to be a $T$-monster if for each Jordan domain $\Omega \subset \mathbb{C}$ and each boundary point $t \in \partial G$, the modified cluster set $R(T f, G, t, \Omega)$ is maximal. The set of all $T$-monsters will be denoted by $\mathcal{M}(T, G)$.

Thanks to the observation by Grosse-Erdmann, a function $f \in H(G)$ is a holomorphic monster in the sense of Luh if and only if it is a $D^{j}$-monster for every $j \in \mathbb{Z}$. In addition, it is also proved in [7] that it suffice to take $\Omega=\mathbb{D}$ in the definition of $T$-monster.

Now, the problem is to determine when an operator $T$ supports a $T$-monster and, in this case, to determine how big is the set of $T$-monsters. In this direction, the next definition is introduced in [7].

Definition 3.5 Let $G$ be an arbitrary open subset of $\mathbb{C}, G \neq \mathbb{C}$. A (not necessarily linear) continuous operator $T: H(G) \rightarrow H(G)$ is said to be strongly omnipresent, when the set $\mathcal{M}(T, G)$ of $T$-monsters is residual in $H(G)$.

Up to now, we know that every derivative or antiderivative operator of arbitrary order is strongly omnipresent. But it would be desirable to find more operators with this wild behavior. A general study of strongly omnipresent operators have been made by Bernal, Calderón and Grosse-Erdmann in [7, 10,11], where they provide necessary and sufficient conditions on the operator $T$ to be strongly omnipresent and apply them then to furnish several examples of these operators. The next statement collects the main examples of strongly omnipresent operators.

Theorem 3.6 Let $G$ be an open subset of $\mathbb{C}$ with $G \neq \mathbb{C}$.

1. Let $\Phi \in H(\mathbb{C})$ be a nonzero entire function of subexponential type. Then the associated differential operator $\Phi(D)$ is strongly omnipresent.

2. Let $C_{\varphi}$ be the composition operator on $H(G)$ generated by a function $\varphi \in H(G, G)$. Then $C_{\varphi}$ is strongly omnipresent if and only if, for every open set $V$ with $V \cap \partial G \neq$ $\varnothing$, the set $\varphi(V \cap G)$ is not relatively compact in $G$.

In particular, if $\varphi$ is an entire function, then the associated composition operator $C_{\varphi}$ on $H(\mathbb{C})$ is strongly omnipresent if and only if $\varphi$ is non-constant.

3. Let $L_{\varphi}$ be the superposition operator on $H(G)$ defined by a function $\varphi \in H(\mathbb{C})$. Then $L_{\varphi}$ is strongly omnipresent if and only if the operator $L_{\varphi}$ on $H(\mathbb{D})$ has dense range.

4. If $\psi \in H(G)$ is a non-zero function, then the multiplication operator $M_{\psi}$ is strongly omnipresent.

5. Let $T$ be a strongly omnipresent operator and $S$ an onto linear operator. Then $T \circ S$ is strongly omnipresent. In particular, every onto linear operator is strongly omnipresent. 
6. Assume that $G$ is a simply connected domain, $a \in G, N \in \mathbb{N}_{0}, a_{n} \in H(G)$ $(0 \leq n \leq N)$ and that $\varphi: G \times G \rightarrow \mathbb{C}$ is a function holomorphic in both variables. Consider the integral-differential operator $T$ on $H(G)$ defined by

$$
T f(z)=\sum_{n=0}^{N} a_{n}(z) D^{n} f(z)+\int_{a}^{z} f(t) \varphi(z, t) d t \quad(z \in G),
$$

where the integral is taken along any rectificable curve in $G$ joining a to $z$. If either $a_{N}$ or $\phi$ are non identically null, then $T$ is strongly omnipresent.

The concept of strongly omnipresence is related to the existence of a big set (in a topological sense) of functions with maximal modified cluster sets. But we can also tell something about the "algebraic size" of the set of these functions. For this, in [8] the concept of strongly omnipresence is generalized. Before going in, in order to concrete the connection to cluster sets, let us introduce a new kind of functional cluster sets.

Let $G \subset \mathbb{C}$ be a domain, $U \subset \mathbb{C}$ be an arbitrary open subset and $t \in \partial G$. Assume that $\mathcal{T}=\left(\tau_{n}\right)$ is a sequence of affine linear transformations $\tau_{n}(z)=a_{n} z+b_{n}$ with $\tau_{n}(U) \subset G(n \in \mathbb{N}) a_{n} \rightarrow 0$ and $b_{n} \rightarrow t(n \rightarrow \infty)$. By $R_{\mathcal{T}}(f, G, t, U)$ we will denote the set of functions $g: U \rightarrow \mathbb{C}$ satisfying that for every $g \in H(G)$ there exists a strictly increasing sequence $\left(n_{k}\right) \subset \mathbb{N}$ such that $f\left(\tau_{n_{k}}(z)\right) \rightarrow g(z)$ uniformly on compact subsets of $G$.

Roughly speaking, this definition is the functional analogue of the classical cluster set along a (prefixed) non-relatively compact subset of $G$.

Definition 3.7 Let $G$ be a complex domain and $T$ a (not necessarily linear) continuous operator on $H(G)$. $T$ is said to be totally omnipresent if for every $t \in \partial G$ and every sequence of affine linear transformations $\tau_{n}(z)=a_{n} z+b_{n}$ with $\tau_{n}(\mathbb{D}) \subset G(n \in \mathbb{N})$, $a_{n} \rightarrow 0$ and $b_{n} \rightarrow t(n \rightarrow \infty)$, there exists a dense set of functions $f \in H(G)$ satisfying that $R_{\left(\tau_{n}\right)}(T f, G, t, \mathbb{D})=H(\mathbb{D})$.

Observe that, in particular, a totally omnipresent operator has a dense set of holomorphic functions $f$ such that for every $t \in \partial G$ the modified cluster set $R(T f, G, t, \mathbb{D})$ equals $H(\mathbb{D})$. This observation together with the fact that such a modified cluster set is always a $G_{\delta}$ subset allows us to state that every totally omnipresent operator is strongly omnipresent.

To conclude this section, we establish (see [8]) the promised result about the algebraic size of holomorphic monsters and $T$-monsters. Again, the derivative and the antiderivative operators are totally omnipresent.

Theorem 3.8 Let $T$ be a totally omnipresent operator on $H(G)$. Then there exists a dense linear manifold $\mathcal{D} \subset H(G)$ such that $\mathcal{M}(T, G) \supset \mathcal{D} \backslash\{0\}$.

In particular, if $G$ is a simply connected domain, then there exists a dense linear submanifold of $H(G)$ all of whose non-zero members are holomorphic monsters in the sense of Luh.

The proof relies again on techniques taken from hypercyclicity theory. 


\section{Maximality of cluster sets on Banach spaces of holo- morphic functions}

In this final section we are concerned with looking for functions in some classical Banach spaces of $H(\mathbb{D})$ (namely, Hardy and Bergman spaces) with properties of maximality of classical and functional cluster sets.

If $p \in[1, \infty)$ then the Hardy space $H^{p}$ is the class of functions $f \in H(\mathbb{D})$ for which

$$
\|f\|_{H^{p}}:=\sup _{0<r<1}\left(\int_{0}^{2 \pi}\left|f\left(r e^{i \theta}\right)\right|^{p} \frac{d \theta}{2 \pi}\right)^{1 / p}<\infty .
$$

The Bergman space $\mathcal{A}^{p}$ is the class of functions $f \in H(\mathbb{D})$ such that

$$
\|f\|_{\mathcal{A}^{p}}:=\left(\iint_{\mathbb{D}}|f(z)|^{p} d \sigma(z)\right)^{1 / p}<\infty,
$$

where $d \sigma(z)$ is the normalized area measure. Each of them becomes a Banach space when endowed with the norm $\|f\|_{H^{p}}$ and $\|f\|_{\mathcal{A}^{p}}$, respectively. Recall also that $H^{p} \subset \mathcal{A}^{p}$ with dense continuous inclusion. For more information about these spaces see [19] and [20].

If $f$ is in the Hardy space $H^{p}$ then Fatou's theorem asserts that the radial limit $\lim _{r \rightarrow 1^{-}} f\left(r e^{i \theta}\right)$ exists and is finite for almost every $\theta \in[0,2 \pi]$, see [19]. Therefore $C_{\gamma}(f)$ is a singleton for almost every radial curve $\gamma=\left\{r e^{i \theta}: r \in[0,1)\right\}$. However, for Bergman spaces this argument falls down, because there are functions in $\mathcal{A}^{p}$ without radial limits almost everywhere, see [20]. But, though this "boundary stability" of Hardy functions, in 1972 Brown and Hansen provided the next result about wild behavior of functions in $H^{p}(0<p<\infty)$ at every boundary point, see [15, Theorem 3], that in the language of cluster sets reads as follows.

Theorem 4.1 For every $0<p<\infty$, there exists a residual set $M \subset H^{p}$ such that for each $f \in M$ and each $t \in \mathbb{T}$, the cluster set $C(f, t)$ is maximal.

This result only speaks about global cluster sets but Fatou's theorem avoid us to obtain maximality of radial cluster sets. Nevertheless, we could ask whether at least for a prescribed countable family of curves in $\mathbb{D}$ tending to $\mathbb{T}$ the assertion of Theorem 2.13 holds in $H^{p}$. The next result (see [12, Theorem 2.5]) gives a positive answer, even without the restriction $\operatorname{Osc}(\gamma) \neq \mathbb{T}$.

Theorem 4.2 Suppose that $p \in[1, \infty)$ and that $\Gamma$ is a countable collection of curves in $\mathbb{D}$ tending to the boundary. Then there is a dense linear manifold $\mathcal{D}$ in $H^{p}$ such that $C_{\gamma}(f)=\widehat{\mathbb{C}}$ for every $f \in \mathcal{D} \backslash\{0\}$ and every $\gamma \in \Gamma$.

In view of the last theorem, we cannot expect good assertions on maximality of cluster sets along arbitrary curves. Nonetheless, making a link with Theorem 4.1 Section 3, we can ask about the existence of functions in $H^{p}$ or $\mathcal{A}^{p}$ with maximal functional cluster sets, that is to say, holomorphic monsters or $T$-monsters.

By following this line Bernal and Calderón [9] proved a negative result concerning holomorphic monsters. 
Theorem 4.3 There are no holomorphic monsters (in the sense of Luh) in any Bergman space $\mathcal{A}^{p}(1 \leq p<\infty)$, so in any Hardy space $H^{p}(1 \leq p<\infty)$.

The proof of this theorem point out that the antiderivatives are to blame for the existence of holomorphic monsters. So we can expect positive results about the existence of $T$-monsters, at least for differential operators. Using several tools from the modern theory of hypercyclicity (roughly speaking, an operator is said to be hypercyclic if it possesses a dense orbit; see the survey [23]), Bernal and Calderón [9, Theorem 2.5] proved the next statement.

Theorem 4.4 Assume that $1 \leq p<\infty$. Then we have for $X=H^{p}$ or $\mathcal{A}^{p}$, that the set $\{f \in X: f$ is an Id-monster $\}$ is residual in $X$.

In fact, we can also establish the large "algebraic" size of the set of these functions, as the following theorem shows (see [9, Theorem 2.9]).

Theorem 4.5 For each $1 \leq p<\infty$, there exists a dense linear submanifold $\mathcal{D}$ of $H^{p}$ such that each function $f \in \mathcal{D} \backslash\{0\}$ is an Id-monster. Consequently, the same holds for the Bergman space $\mathcal{A}^{p}$.

As we said before, the problem of looking for holomorphic monsters in Hardy or Bergman spaces are the antiderivatives, but it is possible to obtain $T$-monsters for derivative operators. The next result, that concludes the paper, established a more general property.

Theorem 4.6 Let $P(z)$ be a complex non-zero polynomial. Then for each $1 \leq p<\infty$ there exists some $P(D)$-monster in $H^{p}$, hence in $\mathcal{A}^{p}$.

Acknowledgements: The author wish to thank L. Bernal-González and M.C. CalderónMoreno for their useful and helpful comments and remarks. This work has been partially supported by MCYT Grant BFM2003-03893-C02-01, Thematic Network MTM200421420-E and the Plan Andaluz de Investigación de la Junta de Andalucía FQM-127.

\section{References}

[1] L. V. Ahlfors. Complex Analysis 3rd ed. MacGraw-Hill, New York, 1979.

[2] D. H. Armitage. Harmonic monsters. J. Approx. Theory 123 (2003), 110-116.

[3] C.A. Berenstein and R. Gay. Complex analysis and special topics in harmonic analysis. Springer, New York, 1995.

[4] L. Bernal-González. Omnipresent holomorphic operators and maximal cluster sets. Colloq. Math. 63 (1992), 315-322.

[5] L. Bernal-González. Plane sets having dense holomorphic images. Rev. Roum. Math. Pures Appl. 40 (1995), 567-569. 
[6] L. Bernal-González, A. Bonilla, M.C. Calderón-Moreno and J. A. Prado-Bassas. Maximal cluster sets of $L$-analytic functions along arbitrary curves. Constructive Approx., to appear.

[7] L. Bernal-González and M.C. Calderón-Moreno. Holomorphic T-Monsters and strongly omnipresent operators. J. Approx. Theory 104 (2000), 204-219.

[8] L. Bernal-González and M.C. Calderón-Moreno. Dense linear manifolds of monsters. J. Approx. Theory 119 (2002), 156-180.

[9] L. Bernal-González and M.C. Calderón-Moreno. Monsters in Hardy and Bergman spaces. Complex Variables 47 (2002), 373-382.

[10] L. Bernal-González, M.C. Calderón-Moreno and K.-G. Grosse-Erdmann. Strongly omnipresent operators: general conditions and applications to composition operators. J. Aust. Math. Soc. 72 (2002), 335-348.

[11] L. Bernal-González, M.C. Calderón-Moreno and K.-G. Grosse-Erdmann. Strongly omnipresent integral operators. Integr. Equ. Oper. Theory 44 (2002), 397-409

[12] L. Bernal-González, M.C. Calderón-Moreno and J. A. Prado-Bassas. Maximal cluster sets along arbitrary curves. J. Approx. Theory 129 (2004), 207-216.

[13] L. Bernal-González, M.C. Calderón-Moreno and J. A. Prado-Bassas. Simultaneously maximal radial cluster sets. J. Approx. Theory, 135 (2005), 114-124.

[14] A. Boivin, P. M. Gauthier and P. V. Paramonov. Approximation on closed sets by analytic or meromorphic solutions on elliptic equations and applications. Canad. J. Math. 54 (2002), 945-969.

[15] L. Brown and L. Hansen. On the range of $H^{p}$ functions. Pacific J. Math. 42 (1972), $27-32$.

[16] M. C. Calderón-Moreno. Holomorphic differential operators and plane sets with dense image. Complex Variables 47 (2002), 167-176.

[17] M. C. Calderón-Moreno and J. Müller. Universal holomorphic and harmonic functions with additional properties. Acta Math. Hungar. 105 (2004), 1-15.

[18] E.F. Collingwood and A.J. Lohwater. The theory of cluster sets. Cambridge University Press, 1966.

[19] P.L. Duren. Theory of $H^{p}$ spaces. Academic Press, New York, 1970.

[20] P.L. Duren and A. Schuster. Bergman Spaces. American Mathematical Society, Providence, RI, 2004.

[21] D. Gaier. Lectures on complex approximation. Birkhauser, Basel-LondonStuttgart, 1987. 
[22] K.-G. Grosse-Erdmann. Holomorphe Monster und universelle Funktionen. Mitt. Math. Sem. Giessen, 176 (1987), 1-84.

[23] K.-G. Grosse-Erdmann. Universal families and hypercyclic operators. Bull. Amer. Math. Soc. (N.S.) 36 (1999), 345-381.

[24] P. Henrici. Applied and computational complex analysis, vol. 3. J. Wiley, New York, 1986.

[25] J. Horváth. Topological vector spaces and distributions, Vol. 1. Addison-Wesley, Reading, 1966.

[26] A. N. Kanatnikov. Limiting sets along sequences of compacta (Russian). Dokl. Akad. Nauk SSSR 253 (1980), 14-17. English transl. in: Soviet Math. Dokl. 22 (1980), 5-9.

[27] A. N. Kanatnikov. Limit sets of meromorphic functions with respect to sequences of compacta (Russian). Izv. Akad. Nauk SSSR Ser. Mat. 48 (1984), 1196-1213. English transl. in: Math. USSR-Izv. 25 (1985), 501-517.

[28] S. Kierst and E. Szpilrajn. Sur certaines singularités des fonctions analytiques uniformes. Fund. Math. 21 (1933), 267-294.

[29] W. Luh. On Universal functions. Colloq. Math. Soc. János Bolyai 19 (1976), 503511.

[30] W. Luh. Approximation by antiderivatives. Constr. Approx. 2 (1986), 179-187.

[31] W. Luh. Holomorphic monsters. J. Approx. Theory 53 (1988), 128-144.

[32] W Luh. Multiply universal holomorphic functions. J. Approx. Theory 89 (1997), $135-155$.

[33] W. Luh, V. Martirosian and J. Müller. T-universal functions with lacunary power series. Acta Sci. Math. (Szeged) 64 (1998), 67-79.

[34] A. A. Nersesjan. Carleman sets (Russian). Izv. Akad. Nauk Armjan. SSR Ser. Mat. 6 (1971), 465-471. English transl. in: Amer. MAth. Soc. Transl. 122 (1984), 99-104.

[35] K. Noshiro. Cluster sets. Springer-Verlag, Berlin, 1960.

[36] J. C. Oxtoby. Measure and Category, 2nd. ed. Springer, New York, 1980.

[37] P. Painlevé. Leçons sur la théorie analytique des équations différetielles professées à Stockholm 1895. Hermann, Paris, 1897.

[38] W. Rudin. Real and Complex Analysis, 3rd ed. McGraw-Hill, London, 1987.

[39] I. Schneider. Schlichte Funktionen mit universellen Approximationseigenschaften. Mitt. Math. Sem. Giessen 230 (1997), 1-72. 
[40] R. Tenthoff. Universelle holomorphe Funktionen mit vorgegeben Approximationswegen. Shaker Verlag, Aachen, 2000.

José A. Prado-Bassas

Departmento de Análisis Matemático

Universidad de Sevilla, Apdo. 1160

Avenida de Reina Mercedes

41080 Sevilla, Spain

bassas@us.es 